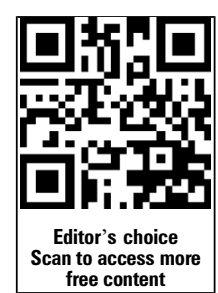

- Additional material is published online only. To view please visit the journal online (http://dx.doi.org/10.1136/ heartjnl-2015-308537).

${ }^{1}$ Johns Hopkins Ciccarone Center for the Prevention of Heart Disease, Baltimore, Maryland, USA

${ }^{2}$ Center for Healthcare Advancement \& Outcomes \& Miami Cardiac \& Vascular Institute (MCVI), Baptist Health South Florida, Miami, FL, USA Henry Ford Hospital, Detroit, Michigan, USA

${ }^{3}$ Division of Cardiovascular Medicine, Henry Ford Hospital, Detroit, Michigan, USA ${ }^{4}$ King Saud bin Abdulaziz University for Health Sciences, King Abdullah International Medical Research Center, King ABdulAziz Cardiac Center, Ministry of National Guard, Health Affairs, Saudia Arabia

\section{Correspondence to} Dr Michael J Blaha, Johns Hopkins Ciccarone Center for the Prevention of Heart Disease, 600 N Wolfe St, Carnegie 565A, Baltimore, MD 21287, USA

mblaha1@jhmi.edu

MJB and RKH are co-first authors of this paper.

Received 12 August 2015 Revised 23 November 2015 Accepted 27 November 2015 Published Online First

5 January 2016

\section{SLinked}

- http://dx.doi.org/10.1136/ heartjnl-2015-309008

\section{CrossMark}

To cite: Blaha MJ Hung RK, Dardari Z, et al. Heart 2016;102:431-437.

\title{
Age-dependent prognostic value of exercise capacity and derivation of fitness-associated biologic age
}

\author{
Michael J Blaha, ${ }^{1}$ Rupert K Hung, ${ }^{1}$ Zeina Dardari, ${ }^{1}$ David I Feldman, ${ }^{1}$ \\ Seamus P Whelton, ${ }^{1}$ Khurram Nasir, ${ }^{1,2}$ Roger S Blumenthal, ${ }_{1}^{1}$ Clinton A Brawner, ${ }^{3}$ \\ Jonathan K Ehrman, ${ }^{3}$ Steven J Keteyian, ${ }^{3}$ Mouaz H Al-Mallah ${ }^{3,4}$
}

\section{ABSTRACT}

Objective Given the aging population and prevalence of sedentary behaviour in the USA, we investigated the impact of differences in exercise capacity associated with age on long-term outcomes. We derived fitnessassociated 'biologic age' as a tool to encourage positive lifestyle changes.

Methods This retrospective cohort study included 57085 patients without established coronary artery disease or heart failure (median age 53 years, 49\% women, 29\% black) who underwent clinically-referred treadmill stress testing at the Henry Ford Health System from 1991 to 2009. Patients were followed for $10.4 \pm 5$ and $5.4 \pm 4$ years for all-cause mortality and myocardial infarction (MI), respectively. We calculated hazard ratios associated with exercise capacity by age deciles using Cox regression models, adjusting for demographic and haemodynamic data, medical history, and medication use. Fitness-associated 'biologic age' was derived as the chronologic age with equivalent mortality or MI risk. Results There were 6356 deaths and 1646 Mls during follow-up. Exercise capacity declined with increasing age. Higher exercise capacity was strongly associated with greater survival, with per-MET HR ranging from 0.82 $(95 \% \mathrm{Cl} 0.78$ to 0.86$)$ in patients under 40 years of age, to $0.88(95 \% \mathrm{Cl} 0.87$ to 0.90$)$ in those over 70 years of age. Biologic age varied markedly —up to three decades - within each age decile, and was a stronger predictor of mortality (C-statistic 0.81 vs 0.77 ) and MI (C-statistic 0.72 vs 0.68 ) than chronologic age. Conclusions Higher exercise capacity remained a powerful predictor of survival despite lower average exercise capacity at older ages, reinforcing its importance in patients of all ages. Fitness-associated biologic age was a stronger predictor of survival than chronologic age, and may be a useful clinical tool for facilitating patient discussions regarding the impact of exercise capacity on long-term risk.

\section{INTRODUCTION}

Exercise capacity is a strong and independent predictor of morbidity and mortality in many diverse patient populations, ${ }^{1-6}$ and is a stronger prognostic indicator than many traditional and non-traditional risk factors. ${ }^{6-8}$ Achieving and maintaining higher exercise capacity remains an important consideration in patients of all ages, ${ }^{79} 10$ despite reports of age-related declines in exercise capacity and its prognostic value. ${ }^{11-13}$ Even though the importance of maintaining exercise capacity is well-known, the prevalence of sedentary behaviour in the USA suggests that new methods of encouraging positive lifestyle changes are needed, and would have important public health ramifications given the aging population. ${ }^{14}$

'Biologic ages' have previously been derived utilising other prognostic indicators such as forced expiratory volume in 1 second $\left(\mathrm{FEV}_{1}\right),{ }^{15}$ coronary artery calcium $(\mathrm{CAC}),{ }^{16-19}$ and serum cholesterol. ${ }^{20}$ Notably, utilising biologic age may enable more accurate risk stratification than chronologic age, ${ }^{17-19} 21$ and may improve patient understanding and compliance with important lifestyle changes including smoking cessation. ${ }^{15}{ }^{20}$ However, derivation of fitness-associated biologic age has not been performed, despite exercise capacity being one of the strongest prognostic predictors of survival, ${ }^{6-9}$ and having the clinical potential to counsel against sedentary behaviour that remains a vital target for the prevention of heart disease and mortality. ${ }^{22}$

This study describes the interaction between age and exercise capacity on risk for all-cause mortality and myocardial infarction (MI) in a multi-ethnic cohort of patients clinically referred for exercise testing. We hypothesised that exercise capacity will remain an important predictor of survival. In addition, we sought to calculate the 'fitness-associated biologic age' to potentially enhance risk stratification and promote physical activity.

\section{METHODS}

\section{Study design}

This study is based on data from the Henry Ford ExercIse Testing Project (The FIT Project), a retrospective cohort study aimed at investigating the long-term implications of exercise capacity on cardiovascular outcomes and all-cause mortality. ${ }^{23}$

The FIT Project is unique in its combined use of: (1) directly measured exercise data; (2) retrospective collection of medical history and medication treatment data taken at the time of the stress test; (3) retrospective supplementation of supporting clinical data using the electronic medical record (EMR) and administrative databases; and (4) epidemiologic follow-up for all-cause mortality and select non-fatal outcomes via linkage with a death registry and medical claims files, respectively.

The FIT Project cohort represents a registry of 69885 consecutive patients who underwent physician-referred treadmill stress testing at Henry Ford Health System in metropolitan Detroit, 
Michigan, between 1991 and 2009. ${ }^{23}$ These medical centres are part of a large, vertically-integrated organisation that provides healthcare and offers a managed care insurance plan. Treadmill, medical history, and medication data were collected by exercise physiologists and nurses, and entered at the time of testing into a common clinical reporting tool that directly populated the EMR. Supporting clinical data and follow-up for cardiovascular outcomes were derived from the EMR and administrative databases shared across Henry Ford Health System. Data from The FIT Project was gathered retrospectively, de-identified in the dataset, and approved by the Henry Ford Health System Institutional Review Board.

\section{Study population}

We considered patients from The FIT Project cohort who did not have a baseline history of established coronary artery disease (CAD) or heart failure $(n=58818)$. Patients were further excluded if any covariates of interest were missing $(n=1733)$, leaving a study population of 57085 individuals. Patients were categorised according to age into five age groups: <40, 40-49.9, $50-59.9,60-69.9$, and $\geq 70$ years of age.

\section{Exercise testing}

All patients underwent routine, clinically-referred, symptomlimited treadmill stress testing following the standard Bruce protocol. ${ }^{24}$ For individuals with repeat stress testing, only the results from the first test were considered in the registry. Patients $<18$ years old at the time of stress testing and patients tested using non-Bruce protocol tests were not included in the registry.

In accordance with clinical guidelines, ${ }^{25}$ treadmill testing was terminated at the discretion of the supervising clinician for reasons that included significant arrhythmias, abnormal haemodynamic responses, diagnostic ST-segment changes, exerciselimiting symptoms such as chest pain or shortness of breath, or if the patient was unwilling or unable to continue. Resting heart rate and blood pressure were taken before stress testing by clinical personnel. Exercise capacity, expressed as estimated metabolic equivalents of task (MET), was calculated by the treadmill controller system (Q-Stress, Quinton Instruments, Bothell, Washington, USA) using peak speed and grade-rather than duration-based on equations reported by the American College of Sports Medicine, and was further categorised into four groups for select analyses: $<6,6-9,10-11$, and $\geq 12$ METS.

\section{Medical history and medication use}

A medical history including age, gender, race, indication for testing, risk factor burden, active medication use, and past medical history was obtained by trained nurses and/or exercise physiologists immediately before the stress test. Race was defined exclusively by self-report. Obesity was defined by selfreport and/or assessment by the clinician historian. Current smoking was defined as self-reported active smoking at the time of testing. Family history of CAD was defined as compatible history in a first degree relative. Indication for stress testing was extracted from the stress test requisition provided by the referring physician, and subsequently categorised into common indications (chest pain, shortness of breath, preoperative evaluation, etc).

Information on medication use and past medical history was supplemented by a retrospective search of the EMR, administrative databases, and/or pharmacy claims files from enrollees in the integrated health plan. A database-verified diagnosis was considered present when the appropriate International Statistical Classification of Diseases and Related Health Problems 9th revision (ICD-9) code was present on $\geq 3$ separate encounters within the health system. Diabetes mellitus, hyperlipidaemia, and hypertension were defined as a self-reported prior diagnosis, a database-verified diagnosis, and/or use of medications for each medical condition.

\section{Follow-up and event adjudication}

Mortality was ascertained in April 2013, after federal law changes in 2011 limited reporting of certain deaths by state agencies. $^{26}$ An algorithmic search of the Social Security Death Index (SSDI) Death Master File (DMF) was completed using social security number, first name, last name, and date of birth data.

MIs were ascertained in May 2010 through linkage with administrative claims files from services delivered by the affiliated group practice and/or reimbursed by the health plan. Linkage was performed using the appropriate ICD-9 and Current Procedural Terminology (CPT) codes for MI. To limit bias associated with loss to follow-up, patients were censored for MI outcomes at their last contact with the integrated Henry Ford Health System group practice when ongoing coverage with the health plan could no longer be confirmed.

\section{Statistical analysis}

Baseline age groups were compared using $\chi^{2}$ testing or analysis of variances techniques as appropriate. We constructed separate best-fit equations for men and women comparing exercise capacity to age. Fractional polynomial equations were tested, but did not improve model fit over a linear model derived with interaction terms between age, gender, and a single optimum inflection point at age 55. Parsimonious linear equations are presented due to their simplicity for clinical practice.

Unadjusted cumulative incidence of mortality and MIs was derived from Kaplan-Meier estimates, with point estimates calculated at median follow-up times of 10.4 and 5.4 years for mortality and MI, respectively. Cox regression models were utilised to calculate the hazard ratios (HRs) associated with METS by categories, and as a continuous variable. Models were additionally adjusted for age (as a continuous variable, to limit residual confounding), gender, race, resting heart rate, resting systolic and diastolic blood pressures, history of hypertension, dyslipidaemia, obesity, smoking, diabetes mellitus, family history of $\mathrm{CAD}$, presence of medications used to treat chronic obstructive pulmonary disease (COPD), hypertension or hyperlipidaemia, and indication for stress testing.

We derived fitness-associated biologic age analogously to previously published methodology calculating 'arterial age' from CAC. ${ }^{16}$ Briefly, within each gender and age group, independent linear parametric survival models satisfying the proportional hazards assumption were generated separately for chronologic age and METS achieved. Fitness-associated biologic age for each MET value was then calculated by equating the two hazards, corresponding to the chronologic age in which equivalent risk was observed. Biologic age was determined over the 5th-95th centile range of METS values within each age group to prevent over-extrapolation. This procedure was performed separately for both mortality and MI.

To formally compare the predictive value of chronologic versus biologic age, Cox proportional hazard models were performed separately for both chronologic and biologic age as univariate variables, and as part of the Framingham Risk Score (FRS). The area under the receiver-operating characteristic (ROC) curves for each outcome were compared using standard techniques. Statistical analyses were performed using Stata (V.13.1, 2014; StataCorp, College Station, Texas, USA). 


\section{RESULTS}

\section{Baseline characteristics}

Table 1 shows the baseline characteristics and stress test results for the study population $(\mathrm{n}=57085)$, which included 27714 (49\%) women and 16375 (29\%) blacks. Mean age of the study sample at baseline was $53 \pm 13$ years. Across increasingly older age groups, our population had a progressively higher proportion of women, whites, and patients with a history of diabetes, hyperlipidaemia, and hypertension. Older patients also tended to have higher resting systolic blood pressure, have a family history of CAD, and were more likely referred for stress testing due to shortness of breath and preoperative assessment. Younger patients were more likely to be referred for stress testing due to chest pain. Tobacco use and obesity were most common in the 50-59.9 year age group ( $\mathrm{p}<0.001$ for all covariates).

The study cohort achieved a mean exercise capacity of 9.2 \pm 3 METS, declining progressively from 11.1 \pm 3 METS in patients under 40 years old to $6.3 \pm 2$ METS in patients over 70 years old $(p<0.001)$. Figure 1 shows the predicted MET achieved by age and gender. A steeper decline in exercise capacity was observed beginning most prominently during the sixth decade of life in both men and women. Distribution of MET levels is shown in online supplementary eTable 1.

\section{Risk for mortality and MIs}

Patients were followed for a mean of $10.4 \pm 5$ and $5.4 \pm 4$ years for all-cause mortality and incident MI, respectively. There were 6356 deaths and 1646 MIs during follow-up. Table 2 shows the unadjusted cumulative incidence of mortality and MI at median follow-up. The largest absolute differences in mortality rate were observed in the older age groups.

Figure 2 shows the adjusted HRs for mortality and MI associated with exercise capacity by age group. Online supplementary eTable 2 shows the per-MET HRs with stratification by gender. Higher METS achieved were associated with lower mortality and MI risk in both men and women of all age groups. Notably, older age consistently and progressively attenuated the association between exercise capacity and mortality $\left(\mathrm{P}_{\text {trend }}<0.01\right)$, but not the association between exercise capacity and $\mathrm{MI}\left(\mathrm{P}_{\text {trend }}=0.35\right)$. The association between exercise capacity and mortality was strongest in the younger age groups $\left(\mathrm{P}_{\text {trend }}<0.01\right)$.

\section{Biologic age estimates}

Table 3 and online supplementary eTable 3 show the fitness-associated biologic age estimates for each gender and age group. Higher METS achieved was associated with lower

Table 1 Baseline characteristics and stress test results

\begin{tabular}{|c|c|c|c|c|c|c|c|}
\hline Demographic data & $\begin{array}{l}\text { All patients } \\
(n=57085)\end{array}$ & $\begin{array}{l}\text { Age }<40 \\
(n=7896)\end{array}$ & $\begin{array}{l}\text { Age } 40-49.9 \\
(n=15401)\end{array}$ & $\begin{array}{l}\text { Age 50-59.9 } \\
(n=17 \text { 145) }\end{array}$ & $\begin{array}{l}\text { Age } 60-69.9 \\
(n=10288)\end{array}$ & $\begin{array}{l}\text { Age } \geq 70 \\
(n=6355)\end{array}$ & $p$ Value \\
\hline Age (years) & $53 \pm 13$ & $34 \pm 5$ & $45 \pm 3$ & $55 \pm 3$ & $65 \pm 3$ & $75 \pm 4$ & $<0.001$ \\
\hline Female & $49 \%$ & $43 \%$ & $47 \%$ & $50 \%$ & $51 \%$ & $52 \%$ & $<0.001$ \\
\hline \multicolumn{8}{|l|}{ Race } \\
\hline White & $64 \%$ & $56 \%$ & $61 \%$ & $65 \%$ & $68 \%$ & $71 \%$ & $<0.001$ \\
\hline Black & $29 \%$ & $34 \%$ & $31 \%$ & $28 \%$ & $26 \%$ & $25 \%$ & $<0.001$ \\
\hline Other & $7 \%$ & $10 \%$ & $8 \%$ & $7 \%$ & $6 \%$ & $4 \%$ & $<0.001$ \\
\hline \multicolumn{8}{|l|}{ Haemodynamic data } \\
\hline Resting heart rate (bpm) & $73 \pm 12$ & $74 \pm 13$ & $74 \pm 12$ & $73 \pm 12$ & $72 \pm 12$ & $71 \pm 12$ & $<0.001$ \\
\hline Resting SBP (mm Hg) & $131 \pm 19$ & $123 \pm 16$ & $126 \pm 17$ & $131 \pm 18$ & $137 \pm 19$ & $142 \pm 19$ & $<0.001$ \\
\hline Resting DBP (mm Hg) & $81 \pm 10$ & $79 \pm 11$ & $81 \pm 11$ & $82 \pm 10$ & $82 \pm 10$ & $80 \pm 10$ & $<0.001$ \\
\hline ppMHR achieved & $91 \%$ & $91 \%$ & $91 \%$ & $91 \%$ & $90 \%$ & $90 \%$ & $<0.001$ \\
\hline \multicolumn{8}{|l|}{ Medical history } \\
\hline Hx hypertension & $62 \%$ & $36 \%$ & $51 \%$ & $66 \%$ & $76 \%$ & $83 \%$ & $<0.001$ \\
\hline Hypertension medications & $43 \%$ & $20 \%$ & $32 \%$ & $46 \%$ & $58 \%$ & $65 \%$ & $<0.001$ \\
\hline Hx hyperlipidaemia & $43 \%$ & $23 \%$ & $36 \%$ & $49 \%$ & $54 \%$ & $51 \%$ & $<0.001$ \\
\hline Hx smoking & $41 \%$ & $34 \%$ & $43 \%$ & $45 \%$ & $42 \%$ & $36 \%$ & $<0.001$ \\
\hline $\mathrm{Hx}$ diabetes & $18 \%$ & $9 \%$ & $13 \%$ & $19 \%$ & $25 \%$ & $26 \%$ & $<0.001$ \\
\hline Family history of CAD & $51 \%$ & $51 \%$ & $56 \%$ & $54 \%$ & $48 \%$ & $39 \%$ & $<0.001$ \\
\hline Hx obesity & $23 \%$ & $22 \%$ & $24 \%$ & $27 \%$ & $22 \%$ & $15 \%$ & $<0.001$ \\
\hline COPD medications & $9 \%$ & $8 \%$ & $9 \%$ & $9 \%$ & $9 \%$ & $8 \%$ & 0.10 \\
\hline \multicolumn{8}{|l|}{ Indication for stress testing } \\
\hline Chest pain & $62 \%$ & $69 \%$ & $67 \%$ & $60 \%$ & $57 \%$ & $55 \%$ & $<0.001$ \\
\hline Shortness of breath & $9 \%$ & $5 \%$ & $8 \%$ & $9 \%$ & $11 \%$ & $12 \%$ & $<0.001$ \\
\hline Preoperative & $2 \%$ & $1 \%$ & $1 \%$ & $2 \%$ & $3 \%$ & $5 \%$ & $<0.001$ \\
\hline Other & $27 \%$ & $25 \%$ & $24 \%$ & $29 \%$ & $29 \%$ & $28 \%$ & $<0.001$ \\
\hline \multicolumn{8}{|l|}{ Stress test results } \\
\hline Mean METS achieved & $9.2 \pm 3$ & $11.1 \pm 3$ & $10.2 \pm 3$ & $9.3 \pm 3$ & $7.9 \pm 3$ & $6.3 \pm 2$ & $<0.001$ \\
\hline \multicolumn{8}{|l|}{ METS categories: } \\
\hline$<6$ METS & $13 \%$ & $3 \%$ & $5 \%$ & $9 \%$ & $20 \%$ & $44 \%$ & $<0.001$ \\
\hline 6-9 METS & $27 \%$ & $13 \%$ & $19 \%$ & $28 \%$ & $40 \%$ & $39 \%$ & $<0.001$ \\
\hline 10-11 METS & $37 \%$ & $37 \%$ & $43 \%$ & $42 \%$ & $32 \%$ & $15 \%$ & $<0.001$ \\
\hline$\geq 12$ METS & $23 \%$ & $48 \%$ & $33 \%$ & $21 \%$ & $8 \%$ & $1 \%$ & $<0.001$ \\
\hline
\end{tabular}

Baseline characteristics and stress test results in the total cohort and by age groups. $p$ Value shown for variance across age groups.

bpm, beats/min; CAD, coronary artery disease; COPD, chronic obstructive pulmonary disease; DBP, diastolic blood pressure; Hx, history; METS, metabolic equivalents; ppMHR, per cent predicted maximal heart rate (by 220-age); SBP, systolic blood pressure. 


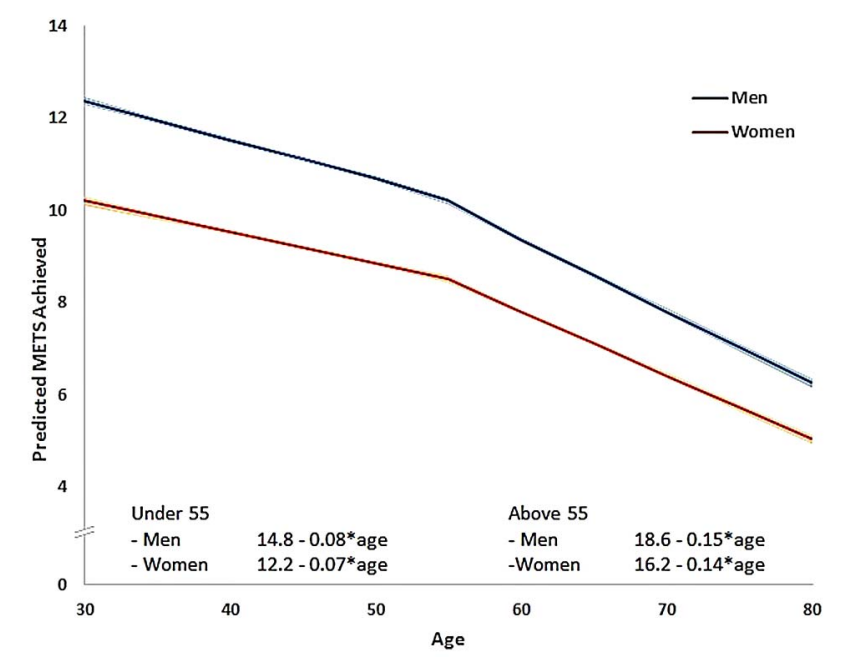

Figure 1 Age- and gender-predicted METS achieved. Predicted METS achieved by age and gender. Formula for predicted METS shown for men and women under and over 55 years old. METS, metabolic equivalents.

biologic age for both mortality and MI, independent of gender and age group. Biologic ages varied widely within each age group, with the smallest range (18 years) observed in men over 70 years and the largest range (38 years) in women under 40 years of age. A 45-year-old woman who achieved 5 vs 13 METS had an equivalent mortality risk of a 60 - vs a 27 -year-old woman, respectively.

Notably in univariate models, biologic age had significantly stronger predictive accuracy compared to chronologic age for mortality (C-statistic: 0.81 vs $0.77, \quad \mathrm{p}<0.001)$ and $\mathrm{MI}$ (C-statistic: 0.72 vs $0.68, \mathrm{p}<0.001)$. When biologic age was substituted for chronologic age in FRS models, similar findings for mortality (C-statistic: 0.72 vs $0.70, \mathrm{p}<0.001$ ) and $\mathrm{MI}$ (C-statistic: 0.71 vs $0.69, \mathrm{p}<0.001$ ) were observed.

\section{Sensitivity analyses}

Sensitivity analyses were performed, excluding those who (1) died within 1 year of their stress test $(n=276)$, (2) underwent stress testing for preoperative cardiac evaluations $(\mathrm{n}=1096)$, and (3) failed to achieve $85 \%$ of their age-predicted maximal heart rate $(n=11053)$. We further adjusted for (4) the decade in which each stress test took place to account for improvements in management of chronic diseases over time, (5) body mass index (BMI) in patients for whom BMI data were collected $(n=36323)$, and (6) baseline $\beta$-blocker therapy to account for potential influences on exercise capacity. ${ }^{27}$ Overall, there were no clinically significant changes to our main results, though mild but statistically significant attenuations were noted in patients on $\beta$-blocker therapy (see online supplementary eTable 4). Online supplementary eTable 5 shows the BMI sensitivity analyses.

\section{DISCUSSION}

Our study has important public health implications for anticipating prognosis in an aging and sedentary population. Higher exercise capacity was associated with lower risk for mortality and MI in patients of all ages, with stronger associations between exercise capacity and mortality observed in younger patients. Fitness-associated biologic age was a stronger predictor of mortality and MI than chronologic age in our cohort. This information may enhance patient understanding of their fitnessmediated risk, and encourage physical activity and other positive lifestyle changes.

\section{Risk for mortality and MI}

Despite lower exercise capacity observed with older age, exercise capacity remained inversely associated with risk for mortality and MI in all patients independent of age. The relative association of exercise capacity with mortality diminished with older age $\left(\mathrm{P}_{\text {interaction }}<0.001\right)$, likely reflecting the greater mortality burden from competing causes in older patients. The per-MET mortality risk reductions in our study-ranging from $18 \%$ in the youngest patients to $12 \%$ in the oldest patientswere similar to observations in other more limited populations, ${ }^{9}{ }^{12}$ and notably were greater than those reported through non-exercise-based models. ${ }^{13}$ We observed per-MET MI risk reductions of approximately $12 \%$ in our cohort without significant effect modification of MI risk from age, though this latter finding may be secondary to insufficient power in our cohort.

Overall, exercise capacity remained among the strongest predictors of both overall and MI-free survival in all patients, reinforcing its continued importance in clinical practice as a modifiable risk factor. ${ }^{6-8}$ Patients often ask "How much fitness is enough?". Our data suggest that a reasonable initial goal is to

Table 2 Unadjusted cumulative incidence of mortality and MI

\begin{tabular}{|c|c|c|c|c|}
\hline & $<6$ METS & 6-9 METS & 10-11 METS & $\geq 12$ METS \\
\hline \multicolumn{5}{|l|}{ Mortality } \\
\hline$<40$ & $17 \%(12 \%$ to $23 \%)$ & $4 \%(3 \%$ to $6 \%)$ & $1 \%(1 \%$ to $2 \%)$ & $1 \%(1 \%$ to $1 \%)$ \\
\hline 40-49.9 & $14 \%(12 \%$ to $17 \%)$ & $4 \%(4 \%$ to $5 \%)$ & $2 \%(2 \%$ to $3 \%)$ & $2 \%(1 \%$ to $2 \%)$ \\
\hline $50-59.9$ & $16 \%(14 \%$ to $18 \%)$ & $6 \%(5 \%$ to $7 \%)$ & $4 \%(4 \%$ to $5 \%)$ & $3 \%(2 \%$ to $4 \%)$ \\
\hline $60-69.9$ & $27 \%$ ( $25 \%$ to $29 \%)$ & $13 \%(12 \%$ to $14 \%)$ & $8 \%(7 \%$ to $9 \%)$ & $6 \%(5 \%$ to $9 \%)$ \\
\hline$\geq 70$ & $42 \%$ ( $40 \%$ to $44 \%)$ & $27 \%$ ( $25 \%$ to $29 \%)$ & $22 \%(19 \%$ to $25 \%)$ & $11 \%$ (6\% to $23 \%)$ \\
\hline \multicolumn{5}{|l|}{ Ml } \\
\hline$<40$ & $4 \%(2 \%$ to $8 \%)$ & $1 \%(1 \%$ to $2 \%)$ & $1 \%(1 \%$ to $1 \%)$ & $0 \%(0 \%$ to $1 \%)$ \\
\hline 40-49.9 & $3 \%(2 \%$ to $5 \%)$ & $1 \%(1 \%$ to $2 \%)$ & $1 \%(1 \%$ to $1 \%)$ & $1 \%(0 \%$ to $1 \%)$ \\
\hline $50-59.9$ & $3 \%(2 \%$ to $4 \%)$ & $2 \%(2 \%$ to $3 \%)$ & $1 \%(1 \%$ to $1 \%)$ & $1 \%(1 \%$ to $1 \%)$ \\
\hline $60-69.9$ & $5 \%(4 \%$ to $6 \%)$ & $3 \%(2 \%$ to $3 \%)$ & $1 \%(1 \%$ to $2 \%)$ & $1 \%(1 \%$ to $2 \%)$ \\
\hline$\geq 70$ & $7 \%(6 \%$ to $9 \%)$ & $5 \%(4 \%$ to $6 \%)$ & $4 \%(3 \%$ to $6 \%)$ & $5 \%(2 \%$ to $16 \%)$ \\
\hline
\end{tabular}




\section{Mortality}

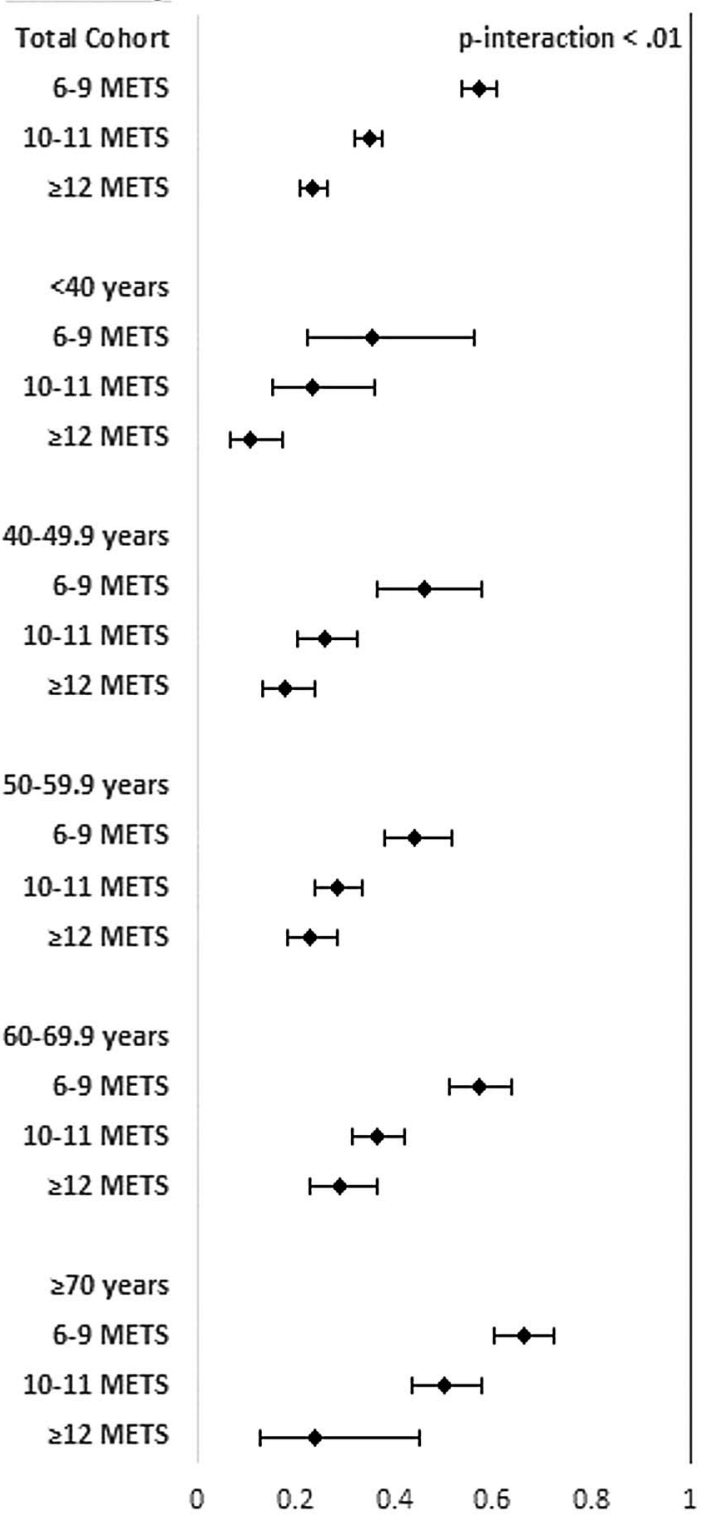

MI

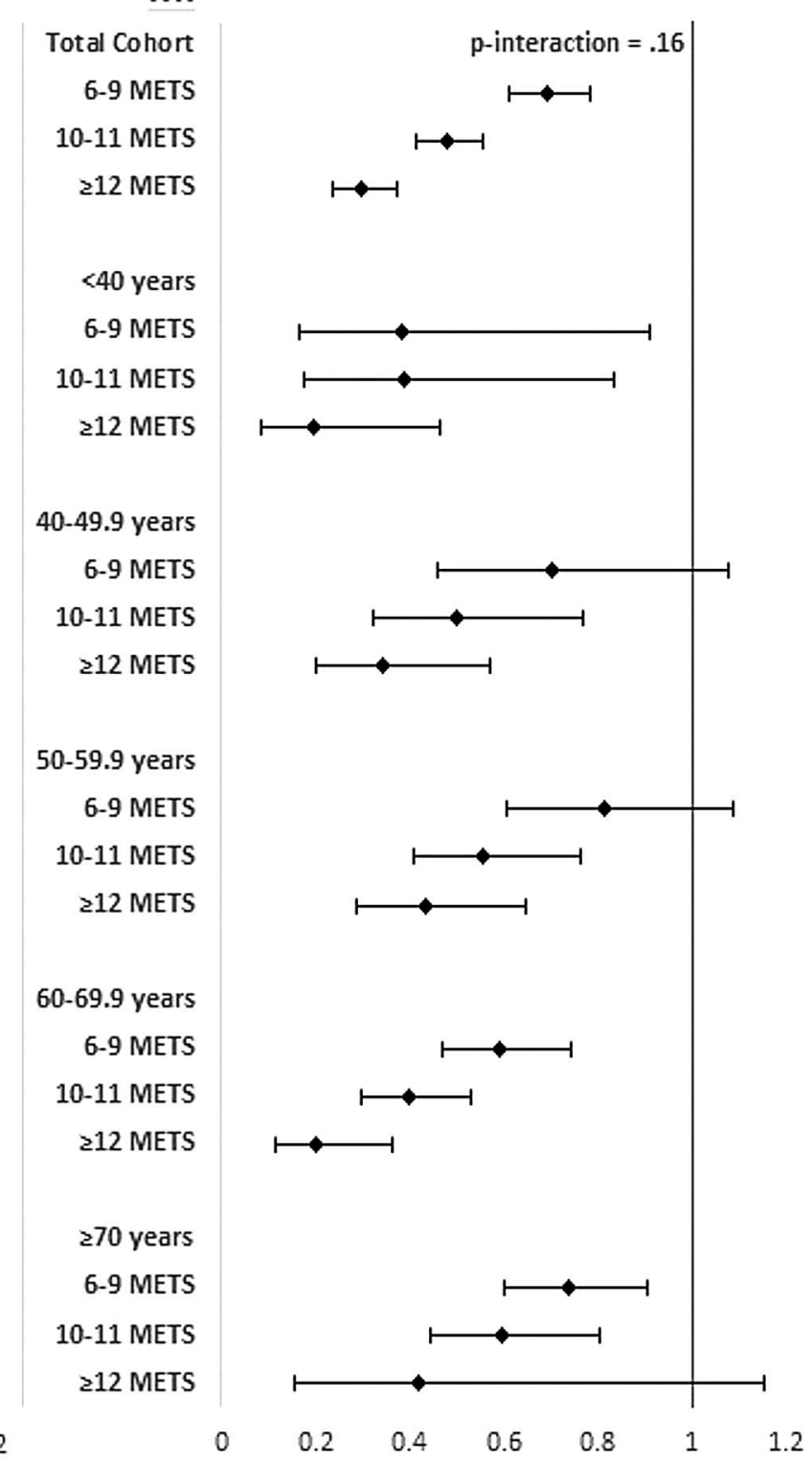

Figure 2 Adjusted hazard ratios for mortality and MI associated with increasing METS categories in the total cohort and by age groups. Per-MET HR available in online supplementary materials. $95 \%$ Cls shown by error bars. P-interaction denotes significance of interaction between age and exercise capacity for each outcome. Notably, older age consistently and progressively attenuated the association between exercise capacity and mortality $\left(P_{\text {interaction }}<0.01\right)$, but not the association between exercise capacity and $\mathrm{Ml}\left(\mathrm{P}_{\text {interaction }}=0.35\right)$. Models adjusted for age, gender, race, resting heart rate, resting systolic and diastolic blood pressure, history of diabetes, hypertension, obesity, smoking, family history of CAD, medications for treatment of hypertension, hyperlipidaemia and COPD, and indication for stress testing. METS, metabolic equivalents; MI, myocardial infarction; $C A D$, coronary artery disease; COPD, chronic obstructive pulmonary disease.

be 'above average' for their age and gender cohort. However, given the observed dose-response relationship between exercise capacity and survival, patients should ultimately be encouraged to be as fit as is practical for their lifestyle and genetics.

\section{Biologic age}

Clinical decision-making for cardiovascular treatment interventions is often guided by risk factor-based algorithms such as the FRS and the Atherosclerotic Cardiovascular Disease (ASCVD) Risk Estimator. ${ }^{28}{ }^{29}$ However, there is substantial heterogeneity in outcomes within chronologic age groups that is not appreciated by these algorithms, which place considerable emphasis on chronologic age as a marker of disease burden such that all older men are classified as 'high-risk' regardless of functional status. The resulting inability to identify 'healthy agers' among the elderly, and 'unhealthy youth', could result in over- and under-treatment in these populations, respectively, ${ }^{18} 19{ }^{21}$ which highlights the clinical need for more accurate risk stratification.

Deriving biologic age from exercise capacity-analogous to 'arterial age' derived from CAC scores-has not been previously described in detail despite its potential clinical utility. ${ }^{16}{ }^{17}$ In our study, fitness-associated biologic age demonstrated greater discrimination for mortality and MI than chronologic age in univariate analyses and when substituted in established risk stratification models such as the FRS. Assessment for biologic age may therefore help identify those who would likely benefit 
Table 3 Biologic age estimates

\begin{tabular}{|c|c|c|c|c|c|c|c|c|c|c|}
\hline \multirow{2}{*}{ Mortality } & \multicolumn{5}{|l|}{ Men } & \multicolumn{5}{|c|}{ Women } \\
\hline & $<40$ & $40-49.9$ & $50-59.9$ & $60-69.9$ & $\geq 70$ & $<40$ & $40-49.9$ & $50-59.9$ & $60-69.9$ & $\geq 70$ \\
\hline 2 METS & & & & & 86 & & & & & 84 \\
\hline 3 & & & & & 84 & & & & 78 & 81 \\
\hline 4 & & & & 77 & 82 & & & & 75 & 79 \\
\hline 5 & & & 67 & 74 & 80 & 60 & 60 & 62 & 71 & 77 \\
\hline 6 & & & 64 & 71 & 77 & 55 & 56 & 59 & 67 & 74 \\
\hline 7 & 55 & 59 & 61 & 68 & 75 & 51 & 52 & 55 & 64 & 72 \\
\hline 8 & 52 & 55 & 58 & 66 & 73 & 46 & 48 & 52 & 60 & 70 \\
\hline 9 & 48 & 51 & 55 & 63 & 71 & 41 & 44 & 48 & 56 & 67 \\
\hline 10 & 44 & 47 & 52 & 60 & 68 & 37 & 39 & 45 & 53 & 65 \\
\hline 11 & 41 & 43 & 49 & 57 & & 32 & 35 & 41 & & \\
\hline 12 & 37 & 39 & 46 & 55 & & 27 & 31 & 37 & & \\
\hline 13 & 33 & 36 & 43 & 52 & & 22 & 27 & 34 & & \\
\hline 14 & 30 & & & & & & & & & \\
\hline 15 & 26 & & & & & & & & & \\
\hline \multirow[t]{2}{*}{ MI } & \multicolumn{5}{|l|}{ Men } & \multicolumn{5}{|c|}{ Women } \\
\hline & $<40$ & $40-49.9$ & $50-59.9$ & $60-69.9$ & $\geq 70$ & $<40$ & $40-49.9$ & $50-59.9$ & $60-69.9$ & $\geq 70$ \\
\hline 2 METS & & & & & 87 & & & & & 82 \\
\hline 3 & & & & & 85 & & & & 83 & 80 \\
\hline 4 & & & & 78 & 83 & & & & 78 & 78 \\
\hline 5 & & & 69 & 74 & 81 & 51 & 63 & 66 & 73 & 76 \\
\hline 6 & & & 66 & 71 & 79 & 47 & 58 & 62 & 68 & 74 \\
\hline 7 & 57 & 61 & 63 & 67 & 76 & 43 & 53 & 57 & 63 & 72 \\
\hline 8 & 52 & 57 & 60 & 64 & 74 & 39 & 48 & 53 & 59 & 70 \\
\hline 9 & 47 & 53 & 56 & 60 & 72 & 35 & 43 & 49 & 54 & 68 \\
\hline 10 & 42 & 49 & 53 & 57 & 70 & 32 & 38 & 44 & 49 & 66 \\
\hline 11 & 37 & 45 & 50 & 53 & & 28 & 33 & 40 & & \\
\hline 12 & 32 & 40 & 47 & 50 & & 24 & 29 & 36 & & \\
\hline 13 & 27 & 36 & 44 & 46 & & 20 & 24 & 31 & & \\
\hline 14 & 22 & & & & & & & & & \\
\hline 15 & 17 & & & & & & & & & \\
\hline
\end{tabular}

the most from pharmacologic and more aggressive lifestyle interventions.

Patients often underestimate the role that fitness plays in determining survival, which may account for variations of up to three decades in our cohort. Utilising biologic age provides an intuitive understanding of fitness-mediated risk and may prompt greater compliance with important lifestyle changes (eg, "you are 45 years old, but your current level of fitness puts you at the same risk for death as a 60-year-old"). In marked contrast, proper interpretation of exercise capacity is non-intuitive due to the lack of clinical consensus on exercise capacity cutoffs, and the need for considering gender differences and age-related declines. $^{13}$

The clinical impact of fitness-associated biologic age has not been studied; however, strategies utilising biologic age derived from other prognosticators have been reported to improve patient understanding and compliance with lifestyle modifications including smoking cessation. ${ }^{15} 20$ Given the prognostic importance of exercise capacity as a modifiable risk factor and the non-intuitive interpretation of exercise capacity, ${ }^{6-9} 13$ determining fitness-associated biologic age may facilitate patient discussions on their fitness-mediated risk, with the goal of promoting physical activity in a sedentary population.

\section{Limitations}

We examined a referral population that is likely enriched in comorbidities compared to healthier cohorts. Our results also may be less generalisable to those at the extremes of age. We lacked data on longitudinal changes in exercise capacity, preventing further assessment for changes in biologic age over time. Key factors that may determine survival such as frailty, substance misuse, diet, physical activity, depression, poor renal function, and other comorbidities may not have been fully accounted for. Cohort effects from changes in secular trends involving smoking, diet, and medical management may have been present but did not significantly alter our findings in sensitivity analyses. Medical history was derived partly through selfreport and presumed indications for medications, which may have been prescribed for other conditions. Sensitivity analyses were conducted to control for confounders from misclassifications in medical history with no significant changes to our results.

Like all cohort studies, a causal relationship between exercise capacity and our outcomes cannot be concluded despite our supportive results. Geographic, selection, or survivorship biases may be present from our inclusion/exclusion criteria. Exercise capacity is determined partly by genetics, which may affect 
individual responses to exercise training. ${ }^{30}$ Furthermore, our results are derived from an initial stress test that may not reflect the patients' true exercise capacity due to symptomatic complaints; however, this approach is representative of what is seen in clinical practice, and therefore is of clinical relevance.

Lastly, our results do not address whether exercise capacity should be routinely measured for risk stratification. Furthermore, additional calibration for biologic age estimates in a primary care setting would be required to generalise our results to a non-referral population. With all tests, the benefits, costs, and risks must be weighed; however, full consideration and discussion of these trade-offs are beyond the scope of this study. Rather, we propose an accessible tool that has the potential to enhance a patient's understanding of their exercise capacity as measured during exercise testing, which is commonly performed in routine clinical practice.

\section{Key messages}

What is already known on this subject?

Exercise capacity is a more central determinant of long-term survival than many traditional cardiovascular risk factors. In other fields of medicine, the concept of 'biologic age' derived from other prognosticators has been shown to improve patient compliance with important lifestyle changes; however, similar derivations of biologic age from exercise capacity have not been performed.

\section{What might this study add?}

We show that exercise capacity remains an important consideration in patients of all ages. We further derive biologic age from exercise capacity, and show that it is a stronger predictor of long-term survival than chronologic age, even when substituted in established risk stratification models such as the Framingham Risk Score.

\section{How might this impact on clinical practice?}

With the derivation of biologic age, a patient's level of exercise capacity may now be intuitively communicated to a patient with the goal of encouraging positive lifestyle changes, for example, "You are 45 years old, but your current level of fitness puts you at the same risk for death and heart attacks as the average person who is 60 years old".

Acknowledgements We thank those at Henry Ford Health Systems who collected and verified data for the registry, and entered and managed data for The FIT Project.

Contributors All authors have made substantial contributions to the conception and design of the study, and acquisition, management, analysis, and/or interpretation of the data, in addition to preparation and review of the present article. MJB and RKH had full access to the study's data and take responsibility for the integrity and accuracy of the data analysis. All authors have approved the article for publication.

Competing interests None declared.

Ethics approval Henry Ford Health Systems IRB.

Provenance and peer review Not commissioned; externally peer reviewed.

\section{REFERENCES}

1 Kodama S, Saito K, Tanaka S, et al. Cardiorespiratory fitness as a quantitative predictor of all-cause mortality and cardiovascular events in healthy men and women: a meta-analysis. JAMA 2009;301:2024-35.
2 Kokkinos PF, Faselis C, Myers J, et al. Interactive effects of fitness and statin treatment on mortality risk in veterans with dyslipidaemia: a cohort study. Lancet 2013;381:394-9.

3 Hung RK, Al-Mallah MH, McEvoy JW, et al. Prognostic value of exercise capacity in patients with coronary artery disease: the FIT (Henry Ford Exerclse Testing) project. Mayo Clin Proc 2014;89:1644-54.

4 Kavanagh T, Mertens DJ, Hamm LF, et al. Prediction of long-term prognosis in 12 169 men referred for cardiac rehabilitation. Circulation 2002;106:666-71.

5 Church TS, LaMonte MJ, Barlow CE, et al. Cardiorespiratory fitness and body mass index as predictors of cardiovascular disease mortality among men with diabetes. Arch Intern Med 2005;165:2114-20.

6 Myers J, Prakash M, Froelicher V, et al. Exercise capacity and mortality among men referred for exercise testing. N Engl J Med 2002;346:793-801.

7 Sui $\mathrm{X}$, LaMonte MJ, Laditka JN, et al. Cardiorespiratory fitness and adiposity as mortality predictors in older adults. JAMA 2007:298:2507-16.

8 Ahmed HM, Al-Mallah MH, McEvoy JW, et al. Maximal exercise testing variables and 10-year survival: fitness risk score derivation from the FIT project. Mayo Clin Proc 2015;90:346-55.

9 Kokkinos P, Myers J, Faselis C, et al. Exercise capacity and mortality in older men: a 20-year follow-up study. Circulation 2010;122:790-7.

10 Berry JD, Willis B, Gupta S, et al. Lifetime risks for cardiovascular disease mortality by cardiorespiratory fitness levels measured at ages 45,55 , and 65 years in men: the Cooper Center Longitudinal Study. J Am Coll Cardiol 2011;57:1604-10.

11 Fleg JL, Morrell CH, Bos AG, et al. Accelerated longitudinal decline of aerobic capacity in healthy older adults. Circulation 2005;112:674-82.

12 Kokkinos P, Faselis C, Myers J, et al. Age-specific exercise capacity threshold for mortality risk assessment in male veterans. Circulation 2014;130:653-8.

13 Nes BM, Vatten LJ, Nauman J, et al. A simple nonexercise model of cardiorespiratory fitness predicts long-term mortality. Med Sci Sports Exerc 2014;46:1159-65.

14 Arias E. United States life tables, 2009. Natl Vital Stat Rep 2014:62:1-63.

15 Parkes G, Greenhalgh T, Griffin M, et al. Effect on smoking quit rate of telling patients their lung age: the Step2quit randomised controlled trial. $B M J$ 2008:336:598-600

16 McClelland RL, Nasir K, Budoff M, et al. Arterial age as a function of coronary artery calcium (from the Multi-Ethnic Study of Atherosclerosis[MESA]). Am J Cardiol 2009;103:59-63.

17 Tota-Maharaj R, Blaha MJ, Blankstein R, et al. Association of coronary artery calcium and coronary heart disease events in young and elderly participants in the multi-ethnic study of atherosclerosis: a secondary analysis of a prospective, population-based cohort. Mayo Clin Proc 2014;89:1350-9.

18 Amin NP, Martin SS, Blaha MJ, et al. Headed in the right direction but at risk for miscalculation: a critical appraisal of the 2013 ACC/AHA risk assessment guidelines. J Am Coll Cardiol 2014;63(25 Part A):2789-94.

19 Blaha MJ, Silverman MG, Budoff MJ. Is there a role for coronary artery calcium scoring for management of asymptomatic patients at risk for coronary artery disease?: clinical risk scores are not sufficient to define primary prevention treatment strategies among asymptomatic patients. Circ Cardiovasc Imaging 2014:7:398-408; discussion 408.

20 Goldman RE, Parker DR, Eaton CB, et al. Patients' perceptions of cholesterol, cardiovascular disease risk, and risk communication strategies. Ann Fam Med 2006;4:205-12.

21 Nakamura E, Moritani T, Kanetaka A. Biological age versus physical fitness age Eur J Appl Physiol Occup Physiol 1989;58:778-85.

22 Robert H Eckel MDF, John M, et al. 2013 AHA/ACC guideline on lifestyle management to reduce cardiovascular risk: a report of the American College of Cardiology/American Heart Association Task Force on Practice Guidelines. J Am Coll Cardio/ 2014:63:2960-84.

23 The FIT Project Investigators. Rationale and design of the Henry Ford Exercise Testing Project (The FIT Project). Clin Cardiol 2014;37:456-61.

24 Bruce RA, Kusumi F, Hosmer D. Maximal oxygen intake and nomographic assessment of functional aerobic impairment in cardiovascular disease. Am Heart J 1973;85:546-62

25 Gibbons R, Balady G, Bricker JT, et al. ACC/AHA 2002 guideline update for exercise testing: summary article. A report of the American College of Cardiology/American Heart Association Task Force on Practice Guidelines (Committee to Update the 1997 Exercise Testing Guidelines). J Am Coll Cardiol 2002:40:1531-40.

26 Social Security Death Master File. Change in Public Death Master File Records. http://www.ssdmf.com/import-change-dmf.pdf (accessed 23 Jul 2014).

27 Kokkinos P, Chrysohoou C, Panagiotakos D, et al. Beta-blockade mitigates exercise blood pressure in hypertensive male patients. J Am Coll Cardiol 2006:47:794-8.

28 Wilson PWF, D'Agostino RB, Levy D, et al. Prediction of coronary heart disease using risk factor categories. Circulation 1998;97:1837-47.

29 Goff DC, Lloyd-Jones DM, Bennett G, et al. 2013 ACC/AHA guideline on the assessment of cardiovascular risk: a report of the American College of Cardiology/ American Heart Association task force on practice guidelines. J Am Coll Cardiol 2014;63(25 Part B):2935-59

30 Bouchard C, Daw EW, Rice T, et al. Familial resemblance for VO2max in the sedentary state: the HERITAGE family study. Med Sci Sports Exerc 1998;30:252-8. 